\title{
I NHALT
}

M. KOBAYASHI: Polarization Phenomena in Elastic-Proton Scattering . . . . . . . . . . 463

W. DRECHSLER: Heisenberg Equations of Motion in a Nonabelian Gauge Theory. . . . . 489

Die „FORTSCHRITTE DER PHYSIK" dienen der Publikation zusammenfassender Berichte über aktuelle Teilgebiete der Physik. Neben Originalberichten in deutscher oder englischer Sprache werden deutsche Úbersetzungen wichtiger fremdsprachiger Berichte abgedruckt.

Aus dem Inhalt der folgenden Hefte:

A.Z. DUBNICKOVA, G.V. EFIMOV, M. A. IVANOV: Nonlocal Quark Model and Meson Decays L. BÁNYAI, A. ALDEA: Master Equation Approach to the Hopping Transport Theory

A. B. SHVARTSBURG: Geometrical Optics in Non-linear Wave Theory

V. SINGH, A. K. RAINA: Bounds on Form Factors and Propagators

J. KUPSCH, W. RÜHL: On the Quantization of Hydrodynamics

Manuskripte werden nach Vereinbarung mit dem Autor von der Schriftleitung entgegengenommen. 